\title{
Transplantation and diabetes (Transdiab): a pilot randomised controlled trial of metformin in impaired glucose tolerance after kidney transplantation
}

\author{
Basil Alnasrallah ${ }^{1 *}$ (D), Tze Liang Goh¹, Lai Wan Chan ${ }^{1}$, Paul Manley ${ }^{1}$ and Helen Pilmore ${ }^{1,2}$
}

\begin{abstract}
Background: Post transplantation diabetes mellitus (PTDM) is a common and serious complication after renal transplantation with significant morbidity and mortality. Metformin has proven benefits in the general population and might be advantageous in the prevention and management of PTDM.

Methods: Transplantation and Diabetes (Transdiab) is a single-centre, unblinded, pilot randomised controlled trial assessing the feasibility, tolerability and efficacy of metformin after renal transplantation in patients with impaired glucose tolerance (IGT). Participants had an oral glucose tolerance test (OGTT) in the 4-12 weeks posttransplantation; those with IGT were randomised to standard care or standard care and metformin 500 mg twice daily and followed up for 12 months.

Results: Seventy eight patients had an OGTT over 24 months, 25 of them had IGT, of those, 19 patients were randomised, giving a feasibility of recruitment of $24.4 \%$. Ten patients were randomised to metformin and 9 patients to standard care. Tolerability and efficacy was similar between the 2 groups with no serious adverse events. There was no difference in secondary outcomes relating to the metabolic profile.

Conclusions: The use of metformin post renal transplantation appeared feasible and safe. Larger randomised controlled trials (RCTs) are needed to establish and confirm the efficacy and safety of metformin post renal transplantation.
\end{abstract}

Trial registration: Australian New Zealand Clinical Trials Registry ACTRN12614001171606. Date of registration 7/11/ 2014.

Keywords: Kidney transplantation, Diabetic kidney disease, Post transplant diabetes mellitus, Randomised control trial

\section{Background}

End-stage kidney disease (ESKD) is a major public health problem with rising prevalence worldwide. In 2015 , there were 4368 people receiving renal replacement therapy for ESKD in New Zealand. Of these, 1694 (39\%) had a functioning kidney transplant [1]. Renal transplantation remains the optimal choice for managing ESKD as it offers better survival [2, 3], quality of life [4]

\footnotetext{
*Correspondence: polbeas@yahoo.com

${ }^{1}$ Department of Nephrology, Auckland City Hospital, Auckland 1023, New

Zealand

Full list of author information is available at the end of the article
}

and long-term cost [5] compared with dialysis treatment. Despite this, the morbidity and mortality of renal transplant recipients remain considerably higher than that of general population [6], with cardiovascular disease accounting for almost $50 \%$ of deaths in these patients [7, $8]$ and prevalence rates of cardiovascular disease 3-5 times higher than matched general population $[9,10]$.

An important risk factor for this high mortality and morbidity is post transplantation diabetes mellitus (PTDM). This entity has been recognized for many years with the first cases being described as early as 1964 by Thomas Starzl, but it was in 2003 that consensus 
guidelines were published setting forth the diagnosis and management recommendations for new onset diabetes after transplantation (NODAT) [11]. More recently the term NODAT was replaced by PTDM as the earlier terminology implies that diabetes prior to transplantation has been adequately excluded which is impractical and often not the case [12].

PTDM is common post transplantation with reported incidence as high 50\% [13], however, the true incidence is difficult to determine as there is a wide quoted range which pertains mainly to the heterogeneity of the trials in the literature. Variables such as the transplanted organ, the criteria used for diagnosis, testing times and immunosuppressive regimens all play significant roles in this fact $[7,14]$.

A combination of traditional and transplant related risk factors are responsible for this risk of PTDM. Of the traditional risk factors, Impaired Glucose Tolerance (IGT), older age, obesity, genetic predisposition, metabolic syndrome, hepatitis $\mathrm{C}$ infection and unhealthy life style have all been implicated in the development of PTDM [15-17]. Of the transplant related factors, the lifelong use of immunosuppressive medications plays a dominant role due to their deleterious effects on glucose metabolism, namely, corticosteroids, calcineurin inhibitors (CNIs), and mammalian target of rapamycin (mTOR) inhibitors [17].

IGT post transplantation is also a risk factor for developing PTDM with 15\% incidence in 1 year [18] and $27 \%$ over 6 years [19]. Furthermore, IGT is associated with increased mortality and overall graft loss [20, 21].

Metformin proved to be an effective therapy to prevent the development of diabetes mellitus in non-transplant patients with impaired glucose metabolism [22]. However, there is lack of evidence for such intervention in transplant patients. Targeting this group of patients will be important to address their PTDM risk.

\section{Methods}

\section{Research aims}

We undertook a pilot study assessing the feasibility, safety, tolerability and efficacy of metformin over 12 months of follow up in patients with IGT diagnosed early after kidney transplantation. The secondary outcomes measured were changes in lipid profile, weight and cardiovascular events.

\section{Trial registration and ethics approval}

The trial has been registered with Australian New Zealand Clinical Trials Registry (ACTRN12614001171606), date of registration 7/11/2014. Ethical approval has been obtained through the Northern B Health and Disability Ethics Committee of the Ministry of Health in New Zealand. Ethics approval number is $14 / \mathrm{STH} / 129$. We have followed CONSORT guidelines for reporting randomized feasibility trials [23].

\section{Study design}

Transplantation and Diabetes (Transdiab) is a single-centre, parallel-group, unblinded, randomised controlled trial with two arms: an intervention group and a standard care group. The study protocol in detail has been previously published [24], an outline of the study is shown in Fig. 1.

\section{Study setting and participants}

Adult patients receiving a kidney transplant between 30 November 2014 and 30 November 2016 at Auckland City Hospital, Auckland, New Zealand, were considered for enrollment.

Patients were eligible for inclusion if they were 18 years or older, non-diabetic, able to participate in all trial investigations for the 12 month follow-up and able to provide written informed consent.

We excluded patients who had one or more the following: diabetes mellitus at the time of transplant (whether on treatment or not), history of antidiabetic therapy, unable to consent, steroid pulse therapy in the 2 weeks prior to OGTT, pregnancy or breast feeding, estimated glomerular filtration rate (eGFR) $\leq 30 \mathrm{~mL} / \mathrm{min} /$ $1.73 \mathrm{~m} 2$ BSA (body surface area) by the Modification of Diet in Renal Disease (MDRD) formula, current substance abuse (including alcohol) and any major illness or comorbidity that may result in death within 12 months.

\section{Recruitment and randomisation}

All potential participants were identified during their admission for the procedure of kidney transplantation. Consented candidates had an OGTT done within 4-12 weeks after the transplantation. Consented patients had their information collected and stored securely, electronically on a password-protected computer and physically in the research office by the research fellow. Enrolling the participants, generating the allocation sequence, and assigning the groups were carried out by the research fellow.

Patients with a 2-h post-load glucose level between 7.8 and $11.1 \mathrm{mmol} / \mathrm{L}$ on OGTT (75 g anhydrous glucose dissolved in water) were randomized. Randomisation was done using a computer-generated sequence allocation in closed envelopes in blocks of four in a 1:1 ratio to receive either standard care or standard care plus metformin $500 \mathrm{mg}$ twice daily, both groups were followed up for 1 year.

\section{Standard care group}

Participants in the standard care group received routine post-transplant care directed by the renal transplant 


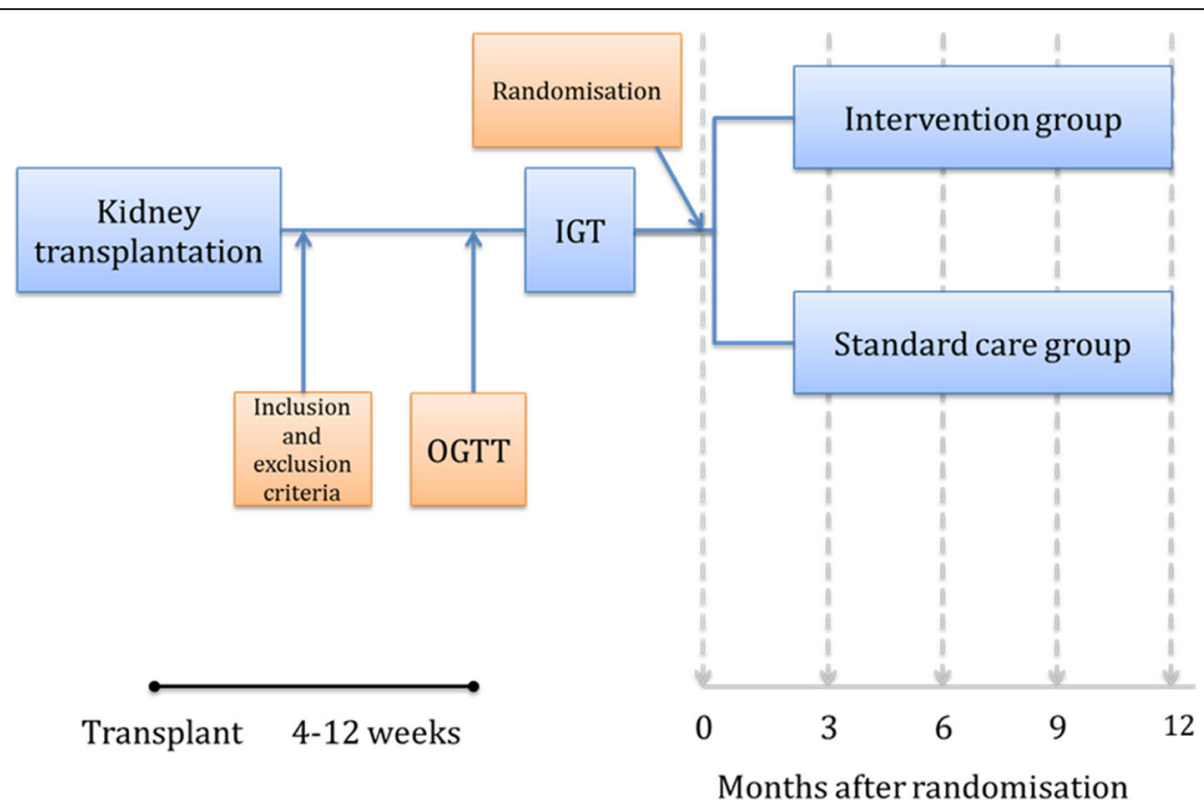

Fig. 1 Flow chart of Transdiab trial. IGT, impaired glucose tolerance; OGTT, oral glucose tolerance test

team. This included immunosuppressive medications and other treatments as per usual local practice. Standard immunosuppression included basiliximab as induction therapy, mycophenolate mofetil $1 \mathrm{~g}$ twice daily, a calcineurin inhibitor (ciclosporin or tacrolimus with dose based on body weight), and steroids (tapering dose over 3 months down to a maintenance of $7.5 \mathrm{mg}$ of prednisone daily).

The lifestyle standard care included advice for regular exercise thrice weekly and a nutritional assessment by a renal dietician. The nutrition care involves guiding patients on the habits of healthy eating and food safety after transplantation.

\section{Intervention group}

Participants randomised to the intervention group received metformin $500 \mathrm{mg}$ twice daily in addition to the standard post-transplant care. The metformin was started at randomisation and continued for 12 months.

\section{Primary outcomes}

\section{Feasibility of recruitment}

Feasibility of recruitment was assessed by examining the percentage of randomised patients of those screened with OGTTs.

\section{Tolerability of metformin}

Tolerability of metformin was evaluated by the gastrointestinal symptom rating scale (GSRS) at 3 and 12 months post randomization. GSRS has been validated to assess symptoms in gastrointestinal disorders [25, 26].

\section{Efficacy of metformin}

Efficacy of metformin was assessed by measuring morning fasting glucose levels and glycated hemoglobin (HbA1c) at 3, 6, 9 and 12 months post randomisation.

\section{Secondary outcomes}

Secondary outcomes included the following: lipid profile at 3, 6, 9 and 12 months; major cardiac events; change in body weight; all adverse events; and proportion of patients who revert to normal glucose metabolism on OGTT at 12 months. The discontinuation or reduction in dose of metformin due to adverse effects was recorded. Serious adverse events were defined as events that were fatal or life-threatening, that resulted in clinically significant or persistent disability, that required or prolonged a hospitalization, or that were judged by the investigator to represent a clinically significant harm to the participant that might require medical or surgical intervention to prevent one of the other events listed above [27].

\section{Statistical analysis}

No formal power calculation was undertaken as this was a feasibility study. Patients were analysed based on their original assignments. The feasibility of recruitment was reported as the percentage of patients screened with OGTT who were randomized to either treatment arm. The second primary outcome, tolerability of metformin, was reported as GSRS at 3 and 12 months, compared between the standard and intervention arms using an analysis of covariance (ANCOVA), and adjusted for GSRS at baseline. The third primary outcome, efficacy of 
metformin, was reported by the levels of $\mathrm{HbAlc}$ and morning blood glucose at 3, 6, 9 and 12 months post randomisation and compared between the two groups. This was done using an ANCOVA adjusted for baseline results.

For categorical variables, the comparisons between groups for significant differences were performed using a chi-square test. All statistical analyses were conducted using the statistical software SPSS (version 24.0). The statistical significance level was set at a probability level of $<0.05$.

\section{Results}

During the 24-month recruitment period, 183 adult patients received a kidney transplant at Auckland City Hospital, Auckland, New Zealand, of which 105 were excluded largely due to pre-existing diabetes mellitus or unwillingness to consent to the study (Fig. 2). Seventy-eight patients had an OGTT, of which 44 (56.4\%) had normal glucose levels, 10 (12.8\%) had PTDM on OGTT and 24 (30.8\%) had IGT. Of those with IGT, 19 were enrolled and randomized with 10 to the intervention arm and 9 to the control arm. Of the remaining 5 patients, 3 withdrew consent before randomisation and 2 were not randomized for clinical reasons, one for deranged liver function tests (LFTs) and the other for a complicated surgical course and concerns for developing acute kidney injury (AKI).

\section{Baseline characteristics}

The intervention group had more females at 6 patients (60\%) compared to the control group with 2 patients (22\%). The duration between transplantation and enrolment was longer in the metformin group at 42.3 (standard deviation (SD) 19.8) days compared to 31.8 (SD 6.8) days in the control group, therefore, the steroids tapering dose was higher in the control group at 17.5 (SD 2.6) $\mathrm{mg}$ compared to 13.9 (SD 3.3) $\mathrm{mg}$ per day in the metformin group. No other significant differences between the 2 groups were identified as per Table 1.

\section{Primary outcomes}

The feasibility of recruitment was $24.4 \%$ with 19 patients recruited of the 78 who underwent OGTT. The tolerability of metformin on GI score was not different between the control and intervention groups at 3 months with scores of 1 (SD 1.6) and 2.3 (SD 2.5) nor at 12 months with scores of 2.3 (SD 4) and 2.9 (SD 1.9), respectively.

The efficacy of metformin on HbAlc and fasting glucose was not different between the 2 groups either at any of the testing points as outlined in Table 2, Figs. 3 and 4.

\section{Secondary outcomes \\ Weight change}

Both groups gained weight by the end of 12 months with the intervention group gaining $2.2 \mathrm{~kg}$ (SD 5.3) and the

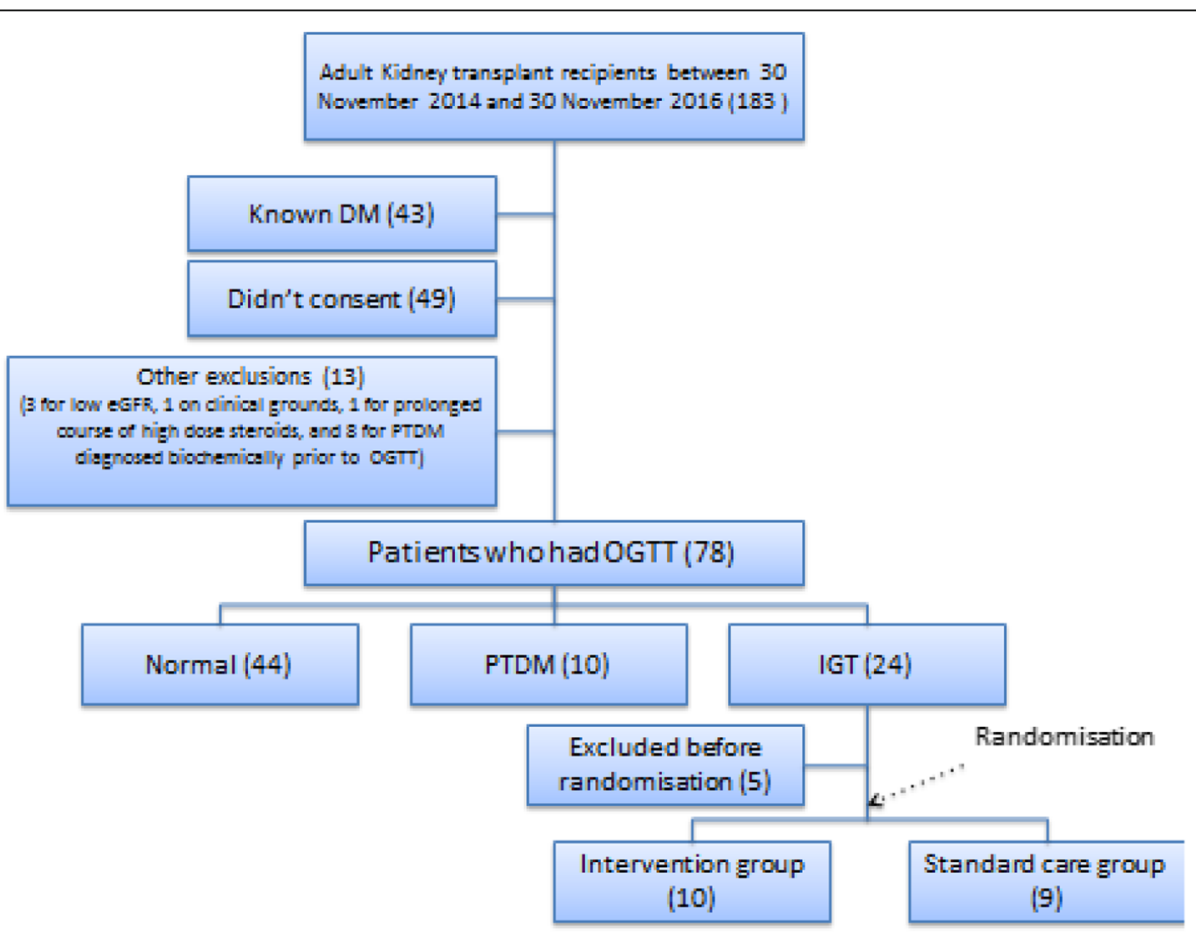

Fig. 2 Flow chart of results of recruitment into Transdiab trial. IGT, impaired glucose tolerance; OGTT, oral glucose tolerance test; PTDM, Post transplantation Diabetes Mellitus; DM, Diabetes Mellitus 
Table 1 Baseline characteristics of participants. eGFR, estimated glomerular filtration rate; HbA1C, glycated hemoglobin; OGT, oral glucose tolerance test, PCR, protein creatinine ratio; TG, triglycerides. NS is not significant when $P$-value is $>0.05$.

\begin{tabular}{|c|c|c|c|}
\hline & Group 1 (Standard care) & Group 2 (Intervention) & $P$-value \\
\hline Number of patients & 9 & 10 & \\
\hline Females & $2(22 \%)$ & $6(60 \%)$ & 0.04 \\
\hline Age (years) & 48.5 (SD 11.6) & 43.4 (SD 9.3) & NS \\
\hline Fasting glucose (mmol/L) & $5.3(\mathrm{SD} 0.7)$ & $5.4(\mathrm{SD} 0.4)$ & NS \\
\hline $2 \mathrm{~h}$ glucose at OGTT (mmol/L) & 9.3 (SD 0.84) & 9.8 (SD 0.7) & NS \\
\hline Body mass index & 28.9 (SD 5.6) & 26.9 (SD 4.8) & NS \\
\hline Hbalc (mmol/mol) & 35.8 (SD 5.5) & 33.8 (SD 3.7) & NS \\
\hline Creatinine (micromol/L) & 136.3 (SD 36.6) & 111.4 (SD 37.9) & NS \\
\hline eGFR in MDRD (ml/min) & $55.1(S D 16.5)$ & 61.4 (SD 20) & NS \\
\hline Gl quality of life & 3.1 (SD 3.4) & $1.4(S D 2.5)$ & NS \\
\hline Serum cholesterol (mmol/L) & $6.2(\mathrm{SD} 1.1)$ & $6.2(\mathrm{SD} 1.2)$ & NS \\
\hline Serum TG (mmol/L) & 2 (SD 0.9) & 2.6 (SD 1) & NS \\
\hline Systolic BP (mm Hg) & 142.3 (SD 16.3) & $125.3($ SD 11.4) & NS \\
\hline Diastolic BP (mm Hg) & 85.3 (SD 8.8) & 78.9 (SD 7.7) & NS \\
\hline Duration between transplant and enrolment & $31.8(S D$ 6.8) & 42.3 (SD 19.8) & 0.001 \\
\hline Urine PCR (mg/mmol) & 36 (SD 22.2) & $53.8(S D$ 35.4) & NS \\
\hline Steroids dose at randomisation (mg) & 17.5 (SD 2.6) & 13.9 (SD 3.3) & 0.024 \\
\hline Number of patients on tacrolimus & $3(33 \%)$ & $6(60 \%)$ & NS \\
\hline
\end{tabular}

"Bold entries have clinical significance"

control group $6.7 \mathrm{~kg}$ (SD 11). However, the difference was not statistically significant $(P$-value 0.12$)$.

\section{Lipid profile}

There was no significant change in the cholesterol and triglycerides (TG) levels between the 2 groups at 3, 6, 9 and 12 months as per the Table 3. Cardiac events: none of the enrolled patients had cardiac events during the follow up.

\section{Adverse events}

One of the 10 patients discontinued metformin 3 months after enrolment due to gastrointestinal symptoms in the form of indigestion and abdominal pain, which resolved after discontinuation. Another patient had a metallic taste in the mouth 6 months after enrolment, the dose of metformin was halved resulting in resolution of symptoms.

One patient in the control group was started on metformin 500 twice daily 6 months after randomisation by

Table 2 Difference between Gl scores, fasting glucose and HbA1c between the 2 groups. Gl,Gastrointestinal. HbA1c, glycated hemoglobin. NS is not significant when $P$-value is $>0.05$.

\begin{tabular}{|c|c|c|c|}
\hline & Group 1 (Standard care) & Group 2 (Intervention) & $P$-value \\
\hline Gl score at & & & All NS \\
\hline 3 months & 1 (SD 1.6) & 2.3 (SD 2.5) & \\
\hline 12 months & 2.3 (SD 4.0) & 2.9 (SD 1.9) & \\
\hline Fasting glucose $(\mathrm{mmol} / \mathrm{L})$ at & & & All NS \\
\hline 3 months & 6.2 (SD 1) & 6 (SD 1.4) & \\
\hline 6 months & 6.1 (SD 1) & 5.8 (SD 1.1) & \\
\hline 9 months & $6.2(S D 0.7)$ & $6(S D 1.1)$ & \\
\hline 12 months & 6 (SD 1.1) & 5.9 (SD 1.5) & \\
\hline $\mathrm{HbA1c}(\mathrm{mmol} / \mathrm{mol})$ at & & & All NS \\
\hline 3 months & 38.9 (SD 4.4) & 41 (SD 7.5) & \\
\hline 6 months & 38.6 (SD 6.9) & 40.1 (SD 6.4) & \\
\hline 9 months & 39.4 (SD 7.7) & 40.3 (SD 5.3) & \\
\hline 12 months & 39.5 (SD 4.6) & 39.4 (SD 6.6) & \\
\hline
\end{tabular}




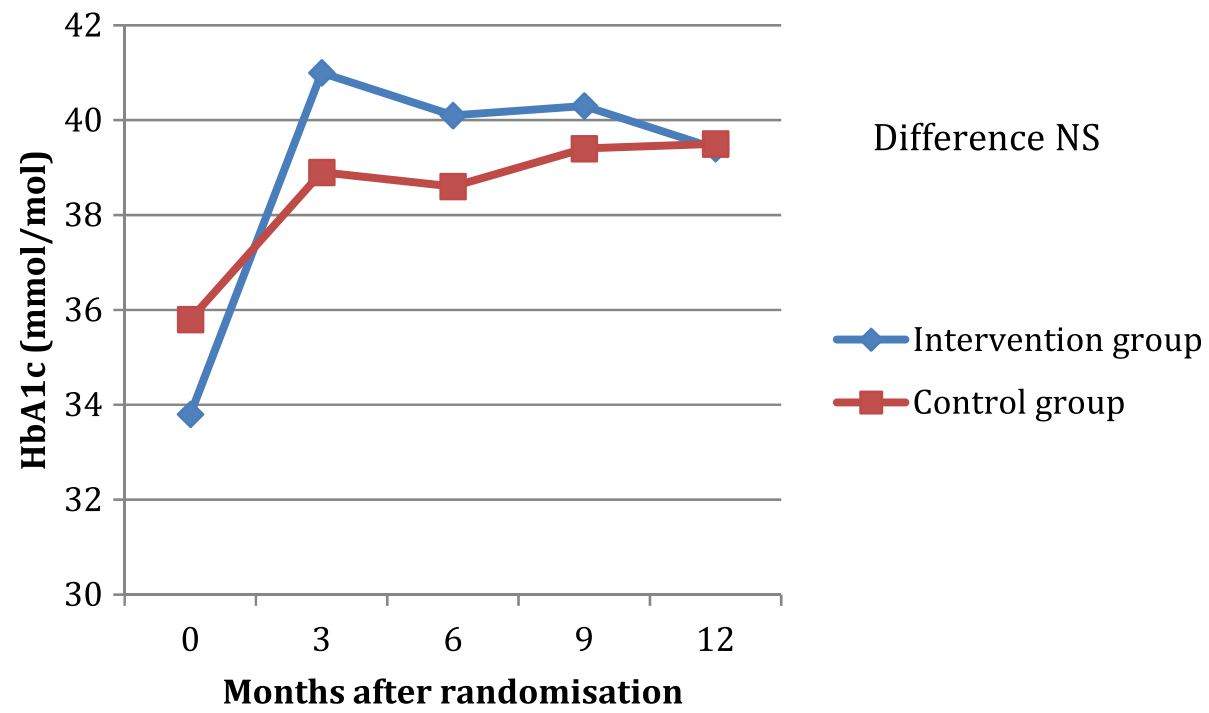

Fig. $3 \mathrm{HbA1c}$ levels during follow up in the intervention and control groups. HbA1c, glycated hemoglobin; NS is not significant when P-value is $>0.05$

the caring physician due to elevated FBG and HbA1c at $7.9 \mathrm{mmol} / \mathrm{l}$ and $54 \mathrm{mmol} / \mathrm{mol}$, respectively.

No patient had a serious adverse drug event and there were no episodes of lactic acidosis.

\section{Patients reverting back to normal glucose metabolism on OGTT}

$60 \%$ of patients in the metformin arm (6 patients) and $22 \%$ in the control arm (2 patients) returned to a normal OGTT at 12 months, ( $p$-value 0.2 ). One patient in each group developed PTDM and 2 had IGT on the 12-month OGTT in each group.

\section{Acute rejection (AR)}

Three of the 19 patients had cellular AR (15.8\%) during the 12 month follow up, 2 in the metformin group and 1 in the control group; no patients had antibody mediated rejection. All cases of rejection were managed successfully with 3 doses of $500 \mathrm{mg}$ IV methylprednisolone and switching the CNI to tacrolimus if the patient was on cyclosporine.

\section{Discussion}

This is the first randomised controlled trial of metformin in patients after kidney transplantation. The addition of the widely available metformin post transplantation will

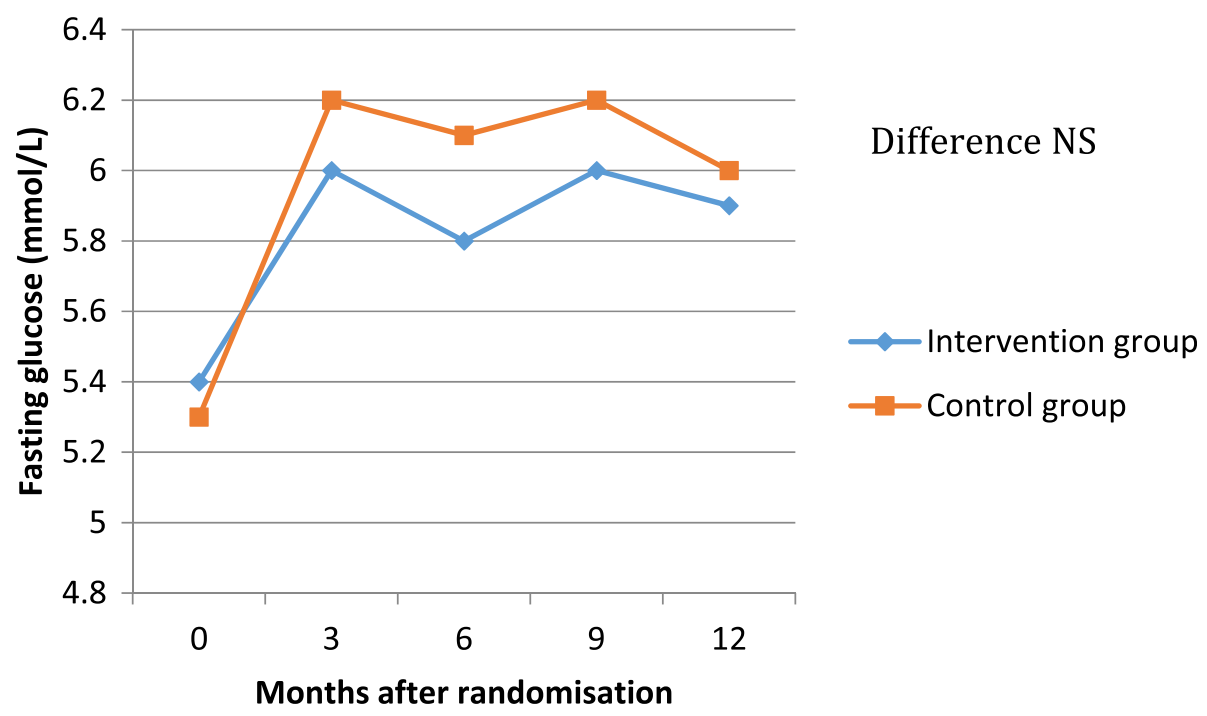

Fig. 4 Fasting glucose levels during follow up in the intervention and control groups. NS is not significant when $P$-value is $>0.05$ 
Table 3 Difference between Lipid profile and weight change between the 2 groups. TG, triglycerides; NS is not significant when Pvalue is $>0.05$

\begin{tabular}{|c|c|c|c|}
\hline & Group 1 (Standard care) & Group 2 (Intervention) & $P$-value \\
\hline Cholesterol $(\mathrm{mmol} / \mathrm{L})$ at & & & All NS \\
\hline 3 months & $5.3(\mathrm{SD} 1)$ & 5.3 (SD 1.6) & \\
\hline 6 months & 4.9 (SD 0.9) & 4.9 (SD 1.1) & \\
\hline 9 months & 4.7 (SD 1) & 5.5 (SD 1.4) & \\
\hline 12 months & 5 (SD 1.2) & 5.6 (SD 1.4) & \\
\hline TG $(\mathrm{mmol} / \mathrm{L})$ at & & & All NS \\
\hline 3 months & $2.1(\mathrm{SD} 1)$ & 2 (SD 1) & \\
\hline 6 months & 2.1 (SD 0.5) & 1.4 (SD 0.7) & \\
\hline 9 months & 2 (SD 0.8) & 1.9 (SD 1.4) & \\
\hline 12 months & 2.3 (SD 1.3) & $2.2(\mathrm{SD} 1.7)$ & \\
\hline Weight change (kilograms) & $+6.7(\mathrm{SD} 11)$ & $+2.2(\mathrm{SD} 5.3)$ & NS \\
\hline
\end{tabular}

be simple and practical for high risk individuals if efficacy is confirmed. We found impaired glucose tolerance in over $30 \%$ of patients undergoing a glucose tolerance test in the first 3 months after kidney transplantation. Of these $79 \%$ were agreeable to participating in a trial of metformin. This indicates that enrolment of patients in studies examining treatments in this sub-group is feasible. Additionally, after excluding patients with diabetes pre-transplant or other exclusion criteria, $61.5 \%$ of potential candidates consented to participation in the study. Forty-nine patients refused to consent. This was largely due to reluctance of patients to take extra medications and the fear of side effects.

Most importantly, there were no significant safety concerns with metformin use early post transplantation in this small group of patients. There were no admissions with lactic acidosis and no significant difference in GI symptoms with the scoring system used. The available safety data for the use of metformin in renal transplant patients is limited. A previous retrospective review by Stephens and colleagues showed that metformin has been used by many renal transplant patients in the United States with no evidence of worse patient or allograft outcomes [28]. Also, a small retrospective study in 2008 reported the safety of metformin in renal transplant patients with PTDM and pre-existing diabetes mellitus [29].

Metformin has been available for many years and has many favourable effects which can be of particular benefit to transplant recipients, namely enhancing glucose metabolism, limiting unwanted weight gain, cardiovascular protection, improving metabolic profile, and anti-neoplastic activity [30]. However, a major deterrent for its use in renal transplant patients has been the safety concern, with physicians fearful of precipitating a dangerous lactic acidosis. Metformin is exclusively renally excreted with a clearance correlating approximately linearly with glomerular filtration rate (GFR) [31].
Renal transplant recipients commonly have abnormal eGFRs with frequent fluctuations in renal function, potentially leading to accumulation of metformin and increasing the risk of lactic acidosis. However, the attitude towards the use of metformin in patients with renal impairment has changed in recent years, as has the perceived risk of lactic acidosis in those patients. Recent large reviews have provided reassurance of the safety of metformin in patients with mild-moderate renal impairment (estimated glomerular filtration rates, $30-60 \mathrm{~mL} /$ min per $1.73 \mathrm{~m} 2$ ), with no significant difference in the rates of lactic acidosis between those treated with or without metformin [32, 33].

Subsequently, more than one regulatory authority has changed its recommendations allowing for the use of metformin in eGFR less than $60 \mathrm{ml} / \mathrm{min}$ and as low as $30 \mathrm{ml} / \mathrm{min}$ [34-36].

In our study, the enrolment was 4 weeks or more after transplant, this allowed for the renal function to stabilize. We also excluded those with eGFRs $<30 \mathrm{mls} /$ min, and educated all enrolled patients about metformin and its potential side effects with action plans when they have health concerns. This allowed for safe application of this intervention, which can be further applied in larger future RCTs.

The other primary outcome, efficacy of metformin, was not statistically different between the 2 groups at any of the designated time points. It would have been challenging to detect a difference with the small sample size. There were no differences in the secondary outcomes between the 2 groups either, and although there was a trend to less weight gain and more patients reverting to normal OGTT at 12 months in the metformin group, this was not statistically significant. Weight gain was noted in both groups, this is a common finding after renal transplantation which can be attributed to a few factors, mainly the use of immunosuppressive medications (such as corticosteroids), 
improvement in well-being and the relaxation of dietary restrictions $[37,38]$.

PTDM is associated with increased rates of cardiovascular disease, cardiovascular death and overall mortality $[7,39,40]$. PTDM is also associated with increased overall graft failure [20, 41].

Therefore, finding effective ways to manage PTDM or even better preventing it will help to address those risks. With the limited evidence on the use of Metformin in kidney transplant patients, it is only possible to use data from the general population to estimate the potential benefit of metformin on glucose metabolism in at risk individuals. The Diabetes Prevention Program Research Group found that metformin reduced the incidence of diabetes in patients with elevated fasting glucose and/or impaired glucose tolerance by $31 \%$ over an average follow up of 2.8 years compared to placebo, with a number needed to treat (NNT) of 13.9 to prevent one case over 3 years [22]. Also, a meta-analysis reported a similar benefit of metformin with a $40 \%$ reduction in the incidence of diabetes mellitus in individuals at high risk over 1.8 years with NNT of 17 [42].

The pathophysiology of the impairment in glucose metabolism appears to be different in PTDM to that in type 2 diabetes mellitus where metformin has been widely used [43,44]. Therefore, the benefits of metformin on glucose metabolism can't be automatically extrapolated and need to be tested in the transplant recipients. The impairment in PTDM is related to a combination of $\beta$-cell dysfunction and insulin resistance [43-45]. This is related primarily to the diabetogenic effects of immunosuppressive medications, as CNIs, which are the cornerstone of contemporary transplant immunosuppressive therapy lead to beta-cell dysfunction and reduction of insulin secretion [46]. Other immunosuppressives lead to different effects, with steroids inducing peripheral insulin resistance and impairing glucose uptake, the exact diabetogenic mechanism of mTOR inhibitors is still uncertain [46].

The main limitation of our study is the small number of patients enrolled. This was expected for a pilot study carried out in a single transplant center, this limited the ability to confidently adjust for some known risk factors for PTDM like tacrolimus use, obesity and older age. Secondly, the dose of metformin was conservative in this trial at $1 \mathrm{~g}$ per day, as the maximal dose is $3 \mathrm{~g}$ per day. As our study is the first to use metformin prospectively in this group of patients, a conservative dose was chosen to encourage participation by patients and physicians and to gather initial safety data before advocating for higher doses in future studies. Using a higher dose of metformin is likely to result in more profound therapeutic effects, which can be entertained in future RCTs.
For future studies, intensive dietary regimens, changing immunosuppression when feasible and using pharmacological therapies are all interventions that could be implemented. Multicenter collaboration should be considered to ensure enrolling adequate number of patients where further stratification can be done, i.e. according to renal function, higher metformin doses, and adjusting for known risk factors. i.e. tacrolimus use. Using OGTT at 1 year as an end point for efficacy would be reasonable as it remains the gold standard for PTDM diagnosis [12], also because the overall rate of PTDM remains largely unchanged beyond 1 year post transplantation [47].

\section{Conclusions}

We have demonstrated reasonable feasibility in a trial using metformin in patients with impaired glucose tolerance after kidney transplantation. In this pilot study the use of metformin in renal transplant recipients with IGT appeared safe and had good tolerability with no serious adverse events, this should help planning future larger RCTs. The efficacy of the use of metformin can be further assessed in studies with adequate number of patients to address the pressing issues related to the prevention and management of PTDM.

\section{Abbreviations \\ AKl: Acute kidney injury; ANCOVA: Analysis of covariance; AR: Acute rejection; BSA: Body surface area; CNIs: Calcineurin inhibitors; eGFR: estimated glomerular filtration rate; ESKD: End stage kidney disease; FBG: Fasting blood glucose; GSRS: Gastrointestinal symptom rating scale; HbA1c: Glycated hemoglobin; IGT: Impaired glucose tolerance; LFTs: Liver function tests; MALA: Metformin associated lactic acidosis; MDRD: Modification of diet in renal disease; mTOR: Mammalian target of rapamycin; NNT: Number needed to treat; NODAT: New onset diabetes after transplantation; OGTT: Oral glucose tolerance test; PCR: Protein creatinine ratio; PTDM: Post transplantation diabetes mellitus; RCT: Randomised controlled trial; SD: Standard deviation; TG: Triglycerides}

\section{Acknowledgments}

Not applicable.

\section{Funding}

The study received a grant from A+ charitable trust in Auckland, New Zealand. The grant covered the salary of the research fellow and also the stationary costs (grant number 6218). The funder had no role in designing or performing the study.

\section{Availability of data and materials}

Data Availability: The data set supporting these findings is not publicly available to protect privacy and confidentiality. Data requests from any interested researcher may be sent to the corresponding author BA: polbeas@yahoo.com

\section{Authors' contributions}

BA participated in the performance of research, data analysis, the writing of the paper and researched data; PM participated in research design and the writing of the paper; TG and LC participated in the performance of the research and reviewed/edited the manuscript; HP participated in research design, the performance of research, and the writing of the paper. All authors have read and approved the final version of the manuscript. 


\section{Ethics approval and consent to participate}

Ethical approval has been obtained through the Northern B Health and Disability Ethics Committee of the Ministry of Health in New Zealand. Ethics approval number is 14/ STH/129. Written informed consent has been obtained from all individuals participating in the study.

\section{Consent for publication}

Not applicable.

\section{Competing interests}

The authors declare that they have no competing interests.

\section{Publisher's Note}

Springer Nature remains neutral with regard to jurisdictional claims in published maps and institutional affiliations.

\section{Author details}

${ }^{1}$ Department of Nephrology, Auckland City Hospital, Auckland 1023, New Zealand. ${ }^{2}$ Department of Medicine, University of Auckland, Auckland, New Zealand.

\section{Received: 18 November 2018 Accepted: 31 March 2019}

Published online: 29 April 2019

\section{References}

1. ANZDATA Registry. The 39th annual ANZDATA report. Australia and New Zealand Dialysis and transplant Registry, Adelaide, Australia. 2017. Available at: http://www.anzdata.org.au/v1/report_2016.html. Accessed 13 Apr 2019.

2. Port FK, Wolfe RA, Mauger EA, et al. Comparison of survival probabilities for dialysis patients vs cadaveric renal transplant recipients. JAMA. 1993;270: 1339-43.

3. McDonald SP, Russ GR. Survival of recipients of cadaveric kidney transplants compared with those receiving dialysis treatment in Australia and New Zealand, 1991-2001. Nephrol Dial Transplant. 2002;17:2212-9.

4. Laupacis A, Keown P, Pus N, et al. A study of the quality of life and costutility of renal transplantation. Kidney Int. 1996;50:235-42.

5. Howard K, Salkeld G, White S, et al. The cost-effectiveness of increasing kidney transplantation and home-based dialysis. Nephrology. 2009;14:123-32.

6. van Walraven C, Manuel DG, Knoll G. Survival trends in ESRD patients compared with the general population in the United States. Am J Kidney Dis. 2014;63:491-9.

7. Hjelmesaeth J, Hartmann A, Leivestad T, et al. The impact of early diagnosed new-onset post-transplantation diabetes mellitus on survival and major cardiac events. Kidney Int. 2006;69:588-95.

8. Pilmore $\mathrm{H}$, Dent $\mathrm{H}$, Chang $\mathrm{S}$, et al. Reduction in cardiovascular death after kidney transplantation. Transplantation. 2010;89:851-7.

9. Baigent C, Burbury K, Wheeler D. Premature cardiovascular disease in chronic renal failure. Lancet. 2000;356:147-52.

10. Foley RN, Parfrey PS, Sarnak MJ. Clinical epidemiology of cardiovascular disease in chronic renal disease. Am J Kidney Dis. 1998;32(5 suppl 3):S112-9.

11. Davidson, J.; Wilkinson, A.; Dantal, J, et al. New-onset diabetes after transplantation: 2003 international consensus guidelines. Transplantation; proceedings of an international expert panel meeting; Barcelona, Spain, 2003; 2003. p. SS3-SS24.

12. Sharif $A$, Hecking $M$, De Vries AP, et al. Proceedings from an international consensus meeting on posttransplantation diabetes mellitus: recommendations and future directions. Am J Transplant. 2014;14(9):19922000 This report summarizes the current consensus recommendations for the management of PTDM and future directions for research.

13. Montori VM, Basu A, Erwin PJ, et al. Posttransplantation diabetes: a systematic review of the literature. Diabetes Care. 2002;25:583-92.

14. Wilkinson A, Davidson J, Dotta F, et al. Guidelines for the treatment and management of new-onset diabetes after transplantation. Clin Transpl. 2005; 19:291-29.

15. Han E, Kim MS, Kim YS, et al. Risk assessment and management of posttransplant diabetes mellitus. Metabolism. 2016;65(10):1559-69.

16. Caillard S, Eprinchard L, Perrin P, Braun L, Heibel F, Moreau F, Kessler L, Moulin $B$. Incidence and risk factors of glucose metabolism disorders in kidney transplant recipients: role of systematic screening by oral glucose tolerance test. Transplantation. 2011;91(7):757-64.
17. Rodrigo E, Fernández-Fresnedo G, Valero R, et al. New-onset diabetes after kidney transplantation: risk factors. J Am Soc Nephrol. 2006;17:S291-5.

18. Hornum $M$, Jørgensen KA, Hansen JM, et al. New-onset diabetes mellitus after kidney transplantation in Denmark. Clin J Am Soc Nephrol. 2010;5:709-16.

19. Hagen M, Hjelmesaeth J, Jenssen T, et al. A 6-year prospective study on new onset diabetes mellitus, insulin release and insulin sensitivity in renal transplant recipients. Nephrol Dial Transplant. 2003;18(10):2154-9.

20. Kasiske BL, Snyder JJ, Gilbertson D, et al. Diabetes mellitus after kidney transplantation in the United States. Am J Transplant. 2003;3:178-85.

21. Valderhaug TG, Hjelmesæth J, Hartmann A, et al. The association of early post-transplant glucose levels with long-term mortality. Diabetologia. 2011; 54(6):1341-9.

22. Knowler WC, Barrett-Connor E, Fowler SE, et al. Diabetes prevention program research group. Reduction in the incidence of type 2 diabetes with lifestyle intervention or metformin. N Engl J Med. 2002;346(6):393-403.

23. Eldridge S, Chan C, Campbell M, et al. CONSORT 2010 statement: extension to randomised pilot and feasibility trials. BMJ. 2016;355:i5239.

24. Alnasrallah B, Pilmore H, Manley P. Protocol for a pilot randomised controlled trial of metformin in pre-diabetes after kidney transplantation: the transplantation and diabetes (Transdiab) study. BMJ Open. 2017;7(8):e016813.

25. Kulich KR, Madisch A, Pacini F, et al. Reliability and validity of the gastrointestinal symptom rating scale (GSRS) and quality of life in reflux and dyspepsia (QOLRAD) questionnaire in dyspepsia: a six country study. Health Qual Life Outcomes. 2008;6:12.

26. Svedlund J, Sjödin I, Dotevall G. GSRS — a clinical rating scale for gastrointestinal symptoms in patients with irritable bowel syndrome and peptic ulcer disease. Dig Dis Sci. 1988;33:129-34.

27. Food and Drug Administration. Code of Federal Regulations. Title 21CFR312. 32a. 2013. http://www.accessdata.fda.gov/scripts/cdrh/cfdocs/cffr/cfrsearch. cfm?fr=312.32. Accessed 13 Apr 2019.

28. Stephen J1, Anderson-Haag TL, Gustafson S, Snyder JJ, Kasiske BL, Israni AK. Metformin use in kidney transplant recipients in the United States: an observational study. Am J Nephrol. 2014;40(6):546-53.

29. B K, Joshi R, Helmuth A. Effectiveness and long-term safety of thiazolidinediones and metformin in renal transplant recipients. Endocr Pract. 2008;14(8):979-84.

30. Sharif A. Should metformin be our Antiglycemic agent of choice posttransplantation? Am J Transplant. 2011;11(7):1376-81.

31. Duong JK, Kumar SS, Kirkpatrick CM, et al. Population pharmacokinetics of metformin in healthy subjects and patients with type 2 diabetes mellitus: simulation of doses according to renal function. Clin Pharmacokinet. 2013; 52:373-84.

32. Salpeter SR, Greyber E, Pasternak GA, et al. Risk of fatal and nonfatal lactic acidosis with metformin use in type 2 diabetes mellitus. Cochrane Database Syst Rev. 201014;(4):CD002967.

33. Inzucchi SE, Lipska KJ, Mayo H, et al. Metformin in patients with type 2 diabetes and kidney disease: a systematic review. JAMA. 2014;312(24):2668-75.

34. National Institute for Health and Care Excellence (NICE). NICE guidelines: the Management of Type 2 diabetes. NICE website. http://www.nice.org.uk/ guidance/cg87/chapter/guidance. Accessed 13 Apr 2019.

35. Canadian Diabetes Association (CDA) Clinical Practice Guideline Pharmacologic Management of Type 2 Diabetes. CDA website. http:// guidelines.diabetes.ca/Browse/Chapter13. Accessed 13 Apr 2019.

36. European Medicines Agency. Metformin and metformin-containing medicines. http://www.ema.europa.eu/ema/index.jsp?curl=pages/medicines/ human/referrals/Metformin_and_metformin-containing_medicines/human_ referral_000397.jsp\&mid=WC0b01ac05805c516f. Accessed 13 Apr 2019.

37. Baum CL, Thielke K, Westin $\mathrm{E}$, et al. Predictors of weight gain and cardiovascular risk in a cohort of racially diverse kidney transplant recipients. Nutrition. 2002;18:139-46.

38. Heaf J, Jakobsen $\mathrm{U}$, Tvedegaard $\mathrm{E}$, et al. Dietary habits and nutritional status of renal transplant patients. J Ren Nutr. 2004;14:20-5.

39. Revanur VK, Jardine AG, Kingsmore DB, et al. Influence of diabetes mellitus on patient and graft survival in recipients of kidney transplantation. Clin Transpl. 2001;15:89-94.

40. Cole $\mathrm{EH}$, Johnston $\mathrm{O}$, Rose $\mathrm{CL}$, et al. Impact of acute rejection and newonset diabetes on long-term transplant graft and patient survival. Clin J Am Soc Nephrol. 2008;3:814-21.

41. Eide IA, Halden TAS, Hartmann A, et al. Associations between Posttransplantation diabetes mellitus and renal graft survival. Transplantation. 2017;101(6):1282-9. 
42. Salpeter SR, Buckley NS, Kahn JA, et al. Meta-analysis: metformin treatment in persons at risk for diabetes mellitus. Am J Med. 2008;121(2):149.

43. Nam JH, Mun Jl, Kim Sl, et al. Beta-cell dysfunction rather than insulin resistance is the main contributing factor for the development of postrenal transplantation diabetes mellitus. Transplantation. 2001;71:1417-23.

44. Hecking M, Kainz A, Werzowa J, et al. Glucose metabolism after renal transplantation. Diabetes Care. 2013;36:2763-71.

45. Zelle DM, Corpeleijn E, Deinum J, et al. Pancreatic beta-cell dysfunction and risk of new-onset diabetes after kidney transplantation. Diabetes Care. 2013; 36:1926-32.

46. Pimentel a AL, Bauer b AC, Camargo JL. Renal posttransplantation diabetes mellitus: an overview. Clin Chim Acta. 2015;450:327-32.

47. Guthoff $M$, Wagner $R$, Weichbrodt $K$, et al. Dynamics of glucose metabolism after kidney transplantation. Kidney Blood Press Res. 2017;42(3):598-607.

Ready to submit your research? Choose BMC and benefit from:

- fast, convenient online submission

- thorough peer review by experienced researchers in your field

- rapid publication on acceptance

- support for research data, including large and complex data types

- gold Open Access which fosters wider collaboration and increased citations

- maximum visibility for your research: over $100 \mathrm{M}$ website views per year

At BMC, research is always in progress.

Learn more biomedcentral.com/submissions 